\title{
Validity of using Single Extruder in Wood Plastic Composite from Rice Straw and High Impact Polystyrene Wastes
}

\author{
Nader Nabil Zaafarani, M.Nour ${ }^{2}$, A. M. El-Kassas ${ }^{3}$ \\ Department of Production Engineering and Mechanical Design, Tanta University \\ Tanta 31527, Egypt \\ Eng.mohammednour@f-eng.tanta.edu.eg
}

\begin{abstract}
This work mainly aims to study the validity of using the single extruder in achieving adhesion and compatibility between rice straw fibers and plastic wastes as an attempt for manufacturing wood plastic composite (WPC). A single extruder was used for mixing recycled high impact polystyrene (RHIPS) and Egyptian natural rice straw (RS) after being fibrated mechanically to produce an eco-friendly synthetic wood that can be named as rice straw plastic composite (RSPC). The components have passed through many operations such as drying, extrusion, shredding, etc.). To produce the final product, the flat press technique was used with a hydraulic press. Samples with $60 \%$ mechanically fibrated rice straw (MFRS) ratio were produced. The density test was operated on the samples according to ASTM-D-792 to reveal the density of the composite. The water absorption was determined according to ASTM-D-570. Using the single extruder for blending the two components didn't achieve high levels of compatibility. However, comparing the results with natural soft, hard wood and medium density fiberboard (MDF) synthetic wood. The prepared RSPC samples exhibited less water absorption and higher density than that of natural and MDF woods.
\end{abstract}

Keywords: Wood plastic composites, rice straw, polystyrene, hot press molding, density.

\section{INTRODUCTION}

Recycled plastics and agriculture wastes are two of the major reasons of global municipal solid waste [1]. Plastics are a major problem due to many reasons. The relatively short life cycle of polymers leads to increasingly consumption of plastic row material and thus high amounts of polymers waste are generated [2]. The non-biodegrability of these wastes pollutes environment and decreases the general human health [3]. From the other hand, depleting trees and forests are also major concerns scaring the global environmental society. Wood wastes are either disposed or burned leading to an extra consumption, depletion and pollution of nature [4]. Several worldwide attempts have been adopted in the developed countries to exploit these types of wastes, especially with the raised needs for alternatives to virgin materials [5]. WPC is an ideal solution to take the advantages of those wastes to produce a new material with desired tailorable properties.

WPCs are a new class of synthetic materials that consist of two main components; a polymeric resin and a cellulosic reinforcing material [6]. They have currently been gaining more and more interest from researchers all over the world as they can divert unexploited wood and plastic wastes from the landfills into construction and building applications that can exist for a long time without deterioration [7]. WPCs have many advantages; high durability, water resistance, Low maintenance, acceptable relative strength and stiffness, resistance to biological and deterioration. Moreover, WPCs are recyclable materials that can give an additional environmental advantage [8].

WPCs are used in outdoor applications where untreated wood products are not suitable [9]. They can be produced to obtain structural building applications including decking sheathings, park benches, roof tiles, fences, landscaping timbers, and window trims [10]. They also can be used for indoor applications such as window and door frames, furniture and floors [11]. WPCs' commercial products have been replacing a variety of products in different applications, especially for construction and outdoor applications [12].

Thus, producing WPCs' products from agricultural and plastic wastes are considered to be a promising solution for recycling the unexploited harmful wastes and divert these wastes into beneficial renewable sources. Therefore, from environmental and economic point of view, the utilization of plastic and rice straw fiber wastes for producing WPCs is a good alternative to natural wood.

The main aim of this work is to study the validity of using the single extruder for manufacturing RSPCs through investigating their water uptake characteristics. Samples with $60 \%$ MFRS fiber ratio were produced. The results were compared to other types of woods.

\section{A. Materials}

As shown in Figure 1, commercial RHIPS in the form of pellets, with a density of $1.05 \mathrm{~g} / \mathrm{cm} 3$ and melt flow index of $11 \mathrm{~g} / 10 \mathrm{~min}(230 \mathrm{C} / 2.16 \mathrm{~kg})$ was used as a matrix. The pellets were sieved using a $3 \mathrm{~mm}$ sieve. Different colors were used to produce multi colors samples.

The reinforcement used for this work was the Egyptian RS wastes. Natural RS was supplied from the Egyptian ministry of agriculture. Then, RS was fibrated mechanically by the attrition process to tear down the wax layer by applying different forces (friction and shear) to the RS chips [13]. The MFRS fibers shown in Figure 2 were sieved using a sieve of 50-mesh size (number of holes per inch ${ }^{2}=50$ ). 


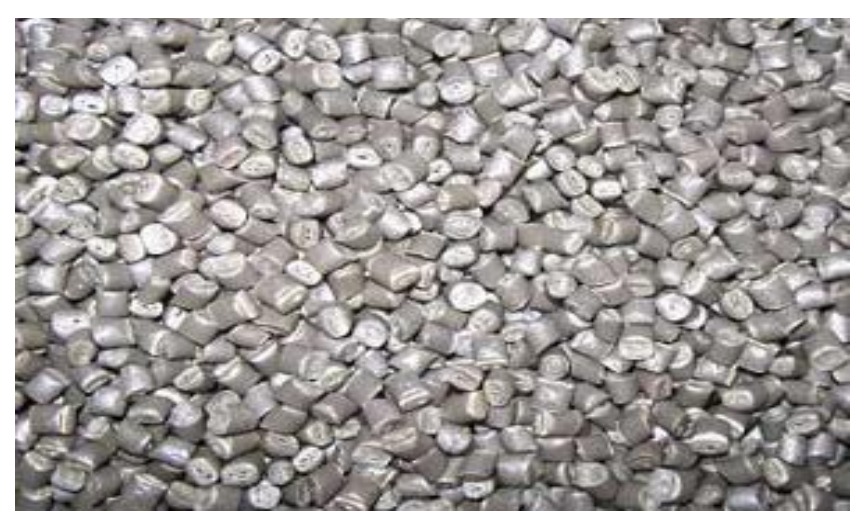

Figure 1 Recycled high impact polystyrene pellets

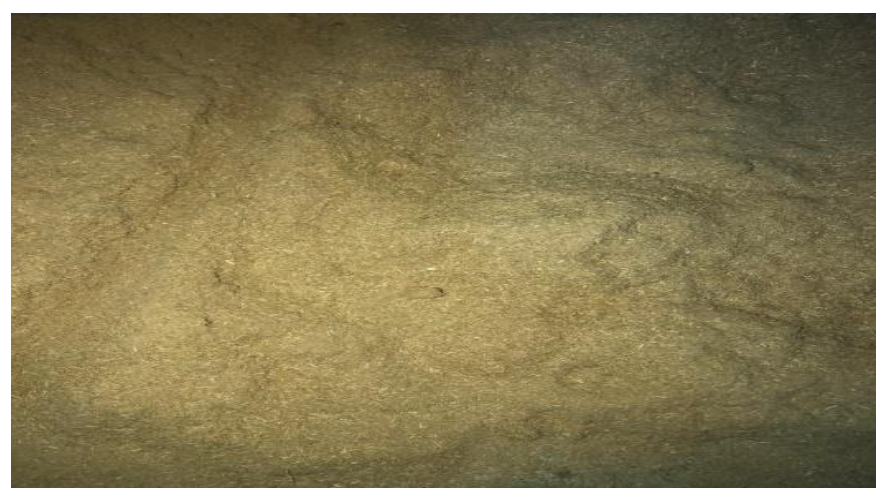

Figure 2 Mechanically fibrated rice straw fibers

\section{B. Composite preparation}

In order to manufacture RSPC products, the raw materials passed through many different steps. This work will demonstrate a sufficient explanation for the processing steps as follows.

\section{Sieving}

Both RHIPS pellets and MFRS fibers were sieved to a chosen size. According to the previous reviews [14], MFRS fibers in 50 mesh and plastic pellets with $3 \mathrm{~mm}$ sizes were used.

\section{Drying}

The two components (RHIPS and MFRS) were dried well using a lab dryer at $100^{\circ} \mathrm{C}$ for 2 hours as in [15]. This step aims to get rid of the existing moisture to reduce the formation of bubbles in the product. Then, they were stored in sealed plastic covers to protect them from moisture in air.

\section{Mixing}

The dried components were weighted according to the required mixing ratio and then they were mixed well using a rotating mixer with a power of $4 \mathrm{hp}$ and $1500 \mathrm{rpm}$ rotating speed for 10 minutes. For this work, composites with $60 \%$ fiber ratio were prepared so as to define the characteristics water uptake resistance. The mixed materials can be shown in Figure 3.

\section{Extrusion}

Blending the components can be done in more than one way, however, in this work an injection molding machine of type Battenfeld shown in Figure 4 was used for blending the two components together using its single extruder. The length over diameter ratio (L/D) of the extruder was 40 and a $30 \mathrm{~mm}$ diameter screw was used with a rotational speed of $50 \mathrm{Rpm}$. Temperatures were 160,170 and $180^{\circ} \mathrm{C}$ over the barrel as shown in Figure 5.

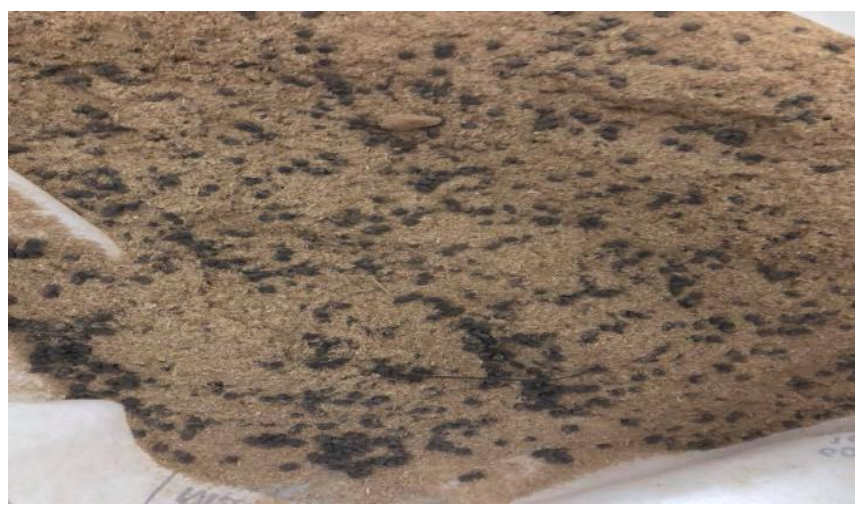

Figure 3 Mixed materials

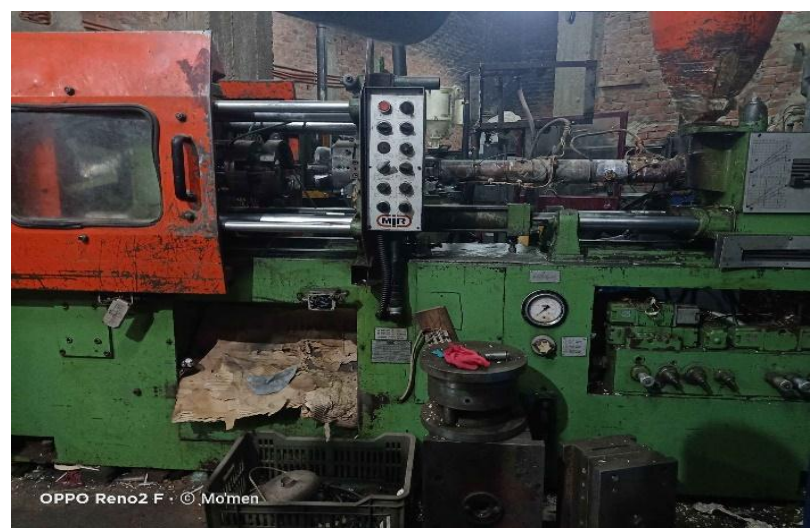

Figure 4 Battenfeld BA202 injection molding machine

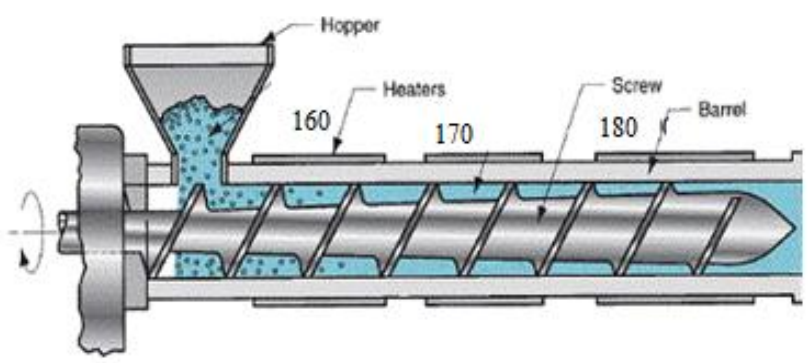

Figure 5 temperatures over the barrel [16]

The mixed materials were put into the injection molding hopper to be blended with the effect of rotation, melting, friction and injection. Figure 6 shows the blended materials using the injection molding machine.

\section{Shredding}

The blended material shown in Figure 6 was shredded using a shredder machine with fixed and rotating knives shown in Figure 7. The shredder machine was with a $10 \mathrm{hp}$ and rotating at $3000 \mathrm{rpm}$. A sieve of $3 \mathrm{~mm}$ size was used in the shredder machine. 


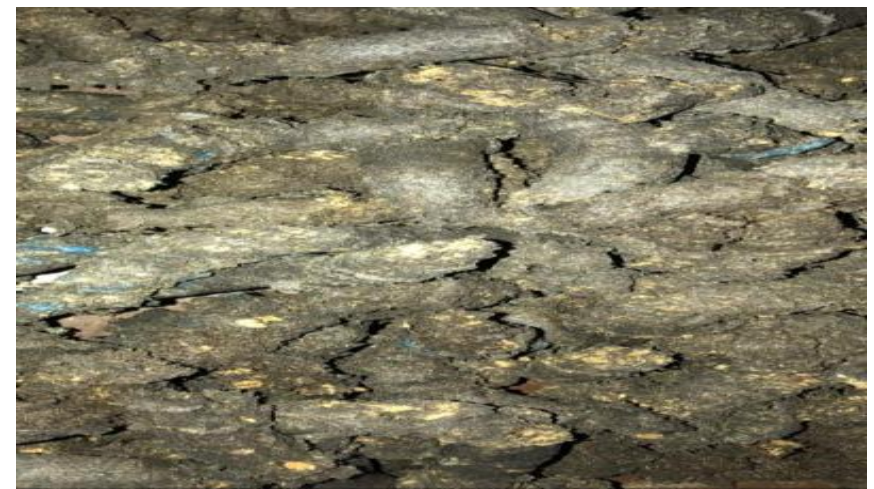

Figure 6 Blended materials with single extruder

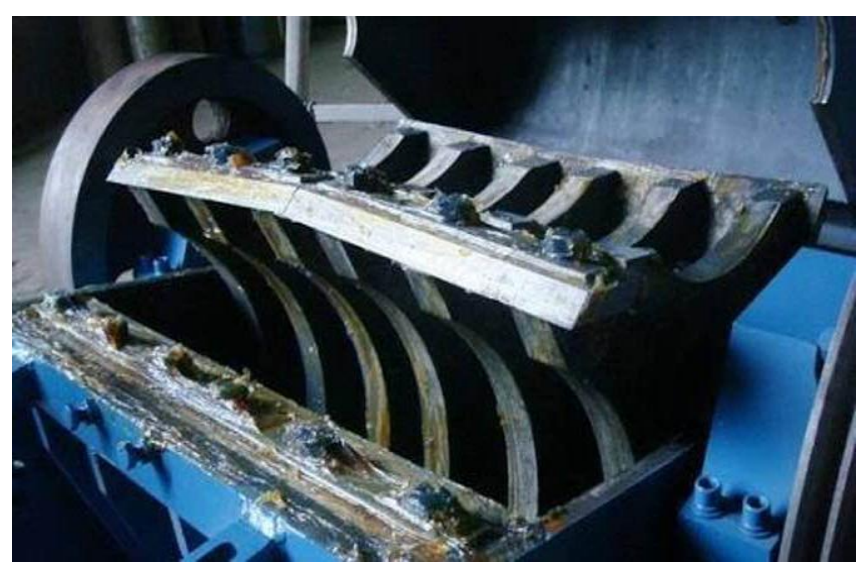

Figure 7 Shredder machine knives

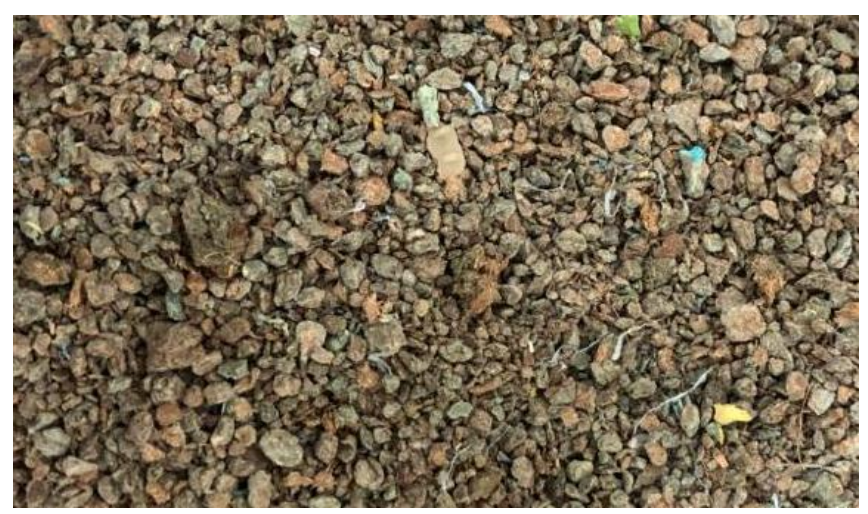

Figure 8 Shredded material with separate fibers

After shredding the blended materials, it was observed that too many fibers were discrete and weren't coated with plastics as shown in Figure 8. This means that blending the two components together with the single extruder didn't achieve high levels of mixing and compatibility.

\section{Pressing}

The shredded extruded materials obtained from last step was formed into a commercial aluminum die $300 \mathrm{~mm} \times 300$ $\mathrm{mm}$ as shown in Figure 9. Upper and lower electrical heaters were attached in the die to melt the mixture.

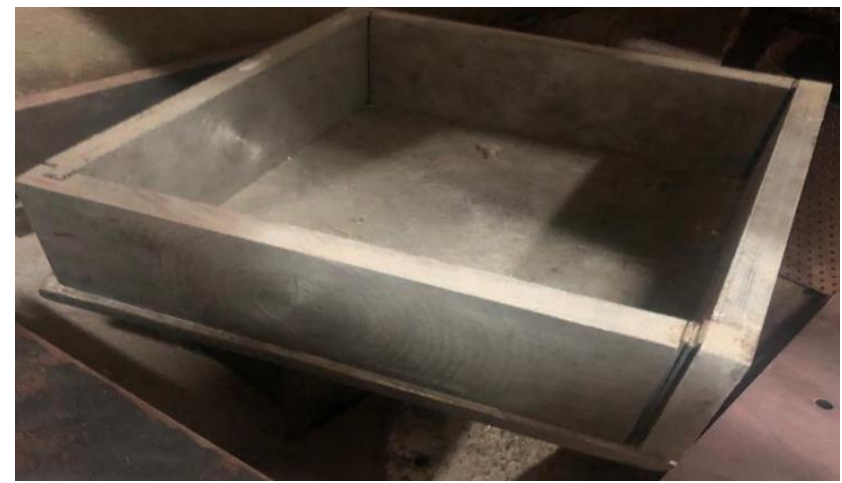

Figure 9 Aluminum die with the frame

Wax paper was used to avoid direct contact. The material was formed into the die then the WPC mats were subjected to hot-pressing using a hydraulic press (Figure 10) using a pressure of 150 bar at temperature of $175^{\circ} \mathrm{C}$ for the both heaters. The specimen was pressed gradually for 10 minutes and then cooled in open air for 1 hour. The final pressed RSPC samples can be shown in Figure 11.

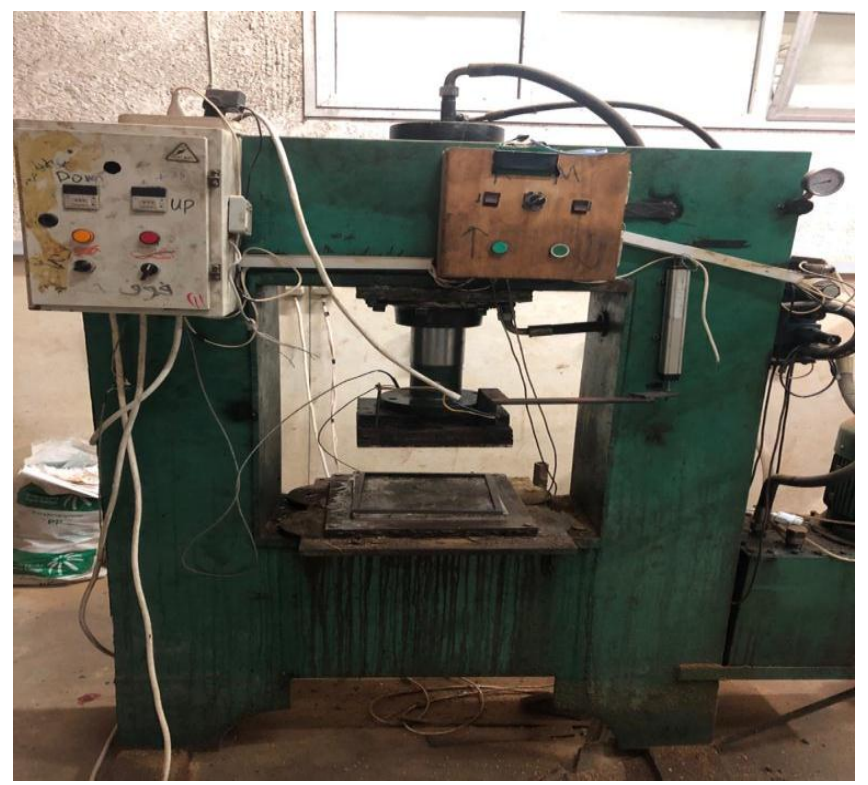

Figure 10 Hydraulic press

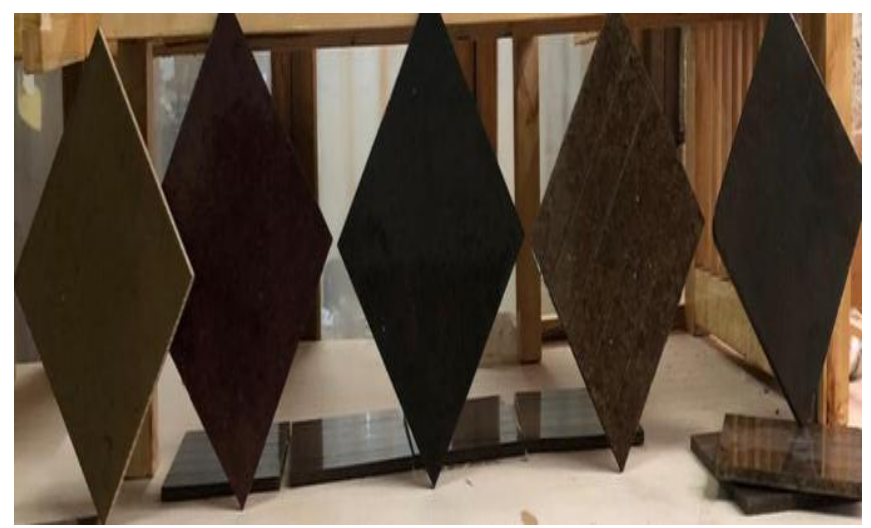

Figure 11 Rice straw plastic composite final product with different colors 


\section{Testing}

\section{Density}

The density of the prepared RSPC samples were measured using displacement method as per ASTM-D-792.

\section{Water absorption}

Water absorption (WA\%) was observed according to ASTM-D-570. The prepared RSPC samples were weighted and recorded before immersing in distilled water for about 24 hours. After that, the samples' surfaces were dried then they were reweighted. The average of the five specimens was calculated.

WA was calculated using the following equation:

$\mathrm{WA} \%=((\mathrm{Ww}-\mathrm{Wd}) * 100) / \mathrm{Wd}[17]$

where,

Ww represents the wet weight of specimen,

$\mathrm{Wd}$ represents the initial dry weight of specimen.

\section{RESULTS AND DISCUSSIONS}

\section{A. Density}

The measured density of the prepared RSPC composite was around $1240 \mathrm{~kg} / \mathrm{m}^{3}$. The densities of the natural hard wood, soft wood, and MDF were 660,470 and $710 \mathrm{~kg} / \mathrm{m}^{3}$ respectively as illustrated in Table 1 . The composite density mainly depends on the densities and specific gravities of the ingredients' interaction in addition to their proportional formulation.

Internal air voids and porosity were developed in RSPC samples. These voids were formed during the hot extrusion process where the moisture existing in the cellulosic fibers (MFRS) forms steam and vapor while plastic decomposition produces a volatile organic compound at high melting temperatures. Similar observations were made by Kazayawoko et al [18]. The density of the developed artificial wood was expected to increase as the input components of the mix are of high density, in addition to the fact that the molding process was under high pressure, which eventually produced a compact structure.

TABLE 1 Physical properties of natural wood, MDF and RSPC [8]

\begin{tabular}{|c|c|c|}
\hline Wood type & Density $\left(\mathbf{k g} / \mathbf{m}^{\mathbf{3}}\right)$ & WA \% \\
\hline Natural hard wood & 660 & 29.2 \\
\hline Natural soft wood & 470 & 42.4 \\
\hline MDF & 710 & 31.9 \\
\hline $60 \%$ fiber loaded RSPC & 1240 & 18.3 \\
\hline
\end{tabular}

B. Water absorption

The prepared RSPC samples recorded high levels of water absorption compared to other WPCs. This is because of the insufficient mixing and compatibility caused by the single extruder. Fibers that weren't coated with plastics absorbed water greatly. However, the WA ability of the prepared RSPC was less than of natural woods and MDF as demonstrated in Figure 12. This is due to the nature of the components specially the plastics. During hot extrusion, plastics melt and encapsulate the MFRS fiber acting as a barrier to resist the water uptake by the fiber content. The water uptake of the hard and soft woods and MDF are higher because most components in natural wood and MDF are lingo-cellulosic and hydrophilic in nature.

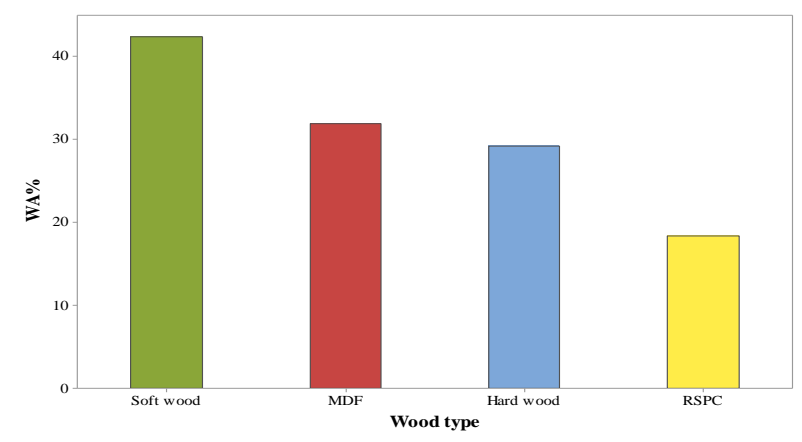

Figure 12 WA\% for natural hard, soft woods, MDF and RSPC

\section{CONCLUSION}

MFRS fibers and RHIPS wastes were recycled to produce WPC flat panels. Panels with different colors were prepared.

The components were mixed using a single extruder. The final composite was manufactured using the hot flat press technique via a hydraulic press.

Using single extruder once for blending the WPC components didn't achieve high levels of compatibility and adhesion. There were lots of fibers separated and not coated with plastics. However, the prepared RSPS samples recorded higher water uptake resistance than natural hard and soft woods and MDF synthetic wood. Thus, the water uptake resistance can be improved using even the twin extruder or the single extruder twice.

The density of the RSPC composite mainly depend on the fiber ratio. Composite $60 \%$ fiber ratio has recorded a density around $1240 \mathrm{~kg} / \mathrm{m}^{3}$.

From a global environmental view, recycling plastics will help to save much more energy and pollution. On the other hand, using rice straw wastes will save trees and forests thus promoting a greener environment. As a cost benefit, these wastes are cheap good raw materials that could be used for the outdoor and construction industry, generating different economical outcomes.

Further study: using the single extruder twice for bending the two components together is suggested for better properties.

\section{REFERENCES}

[1] S. Kazemi Najafi, "Use of recycled plastics in wood plastic composites - A review," Waste Manag., vol. 33, no. 9, pp. 1898 1905, 2013, doi: 10.1016/j.wasman.2013.05.017.

[2] K. L. Pickering, M. G. A. Efendy, and T. M. Le, "A review of recent developments in natural fibre composites and their mechanical performance," Compos. Part A Appl. Sci. Manuf., vol. 83, pp. 98-112, 2016, doi: https://doi.org/10.1016/j.compositesa.2015.08.038.

[3] K. S. Chun et al., "Wood plastic composites made from postused polystyrene foam and agricultural waste," J. Thermoplast. Compos. Mater., vol. 32, no. 11, pp. 1455-1466, 2019, doi: $10.1177 / 0892705718799836$. 
[4] E. Haggar, S. M., and K. Mokhtar, "Wood Plastic Composites," Adv. Compos. Mater. - Anal. Nat. Man-Made Mater., 2011, doi: $10.5772 / 18172$.

[5] M. Schwarzkopf and M. Burnard, "Wood-Plastic CompositesPerformance and Environmental Impacts," 2016, pp. 19-43.

[6] S. B. Eskander and M. E. Tawfik, "Impacts of gamma irradiation on the properties of hardwood composite based on rice straw and recycled polystyrene foam wastes," Polym. Compos., vol. 40, no. 6, pp. 2284-2291, 2019, doi: $10.1002 / \mathrm{pc} .25036$.

[7] M. Kaseem, K. Hamad, F. Deri, and Y. G. Ko, "Material properties of polyethylene/wood composites: A review of recent works," Polym. Sci. - Ser. A, vol. 57, no. 6, pp. 689-703, 2015, doi: 10.1134/S0965545X15070068.

[8] M. A. Binhussain and M. M. El-Tonsy, "Palm leave and plastic waste wood composite for out-door structures," Constr. Build. Mater., vol. 47, pp. 1431-1435, 2013, doi: 10.1016/j.conbuildmat.2013.06.031.

[9] A. Wechsler, S. Hiziroglu, and A. A. Ballerini, "Some of the Properties of Wood Plastic Composites," pp. 1-10, 2008.

[10] M. J. Schwarzkopf and M. Burnard, "Environmental Impacts of Traditional and Innovative Forest-based Bioproducts," Environ. Impacts Tradit. Innov. For. Bioprod., pp. 19-44, 2016, doi: 10.1007/978-981-10-0655-5.

[11] D. J. Gardner, Y. Han, and L. Wang, "Wood-Plastic composite technology," Curr. For. Reports, vol. 1, no. 3, pp. 139-150, 2015, doi: 10.1007/s40725-015-0016-6.

[12] S. K. Yeh, S. Agarwal, and R. K. Gupta, "Wood-plastic composites formulated with virgin and recycled ABS," Compos. Sci. Technol., vol. 69, no. 13, pp. 2225-2230, 2009, doi: 10.1016/j.compscitech.2009.06.007.

[13] A. El-Kassas and A.-H. Mourad, "Novel fibers preparation technique for manufacturing of rice straw based fiberboards and their characterization," Mater. Des., vol. 50, pp. 757-765, Sep. 2013, doi: 10.1016/j.matdes.2013.03.057.

[14] L. W. Gallagher and A. G. Mcdonald, "The effect of micron sized wood fibers in wood plastic composites," Maderas. Cienc. y Tecnol., vol. 15, no. 3, pp. 357-374, Oct. 2013, doi: 10.4067/S0718-221X2013005000028.

[15] E. Saber et al., "Characterization of Plastic Composite Based on HIPS Loaded with Bagasse,” Egypt. J. Chem., vol. 60, no. 6, pp. 1101-1110, Dec. 2017, doi: 10.21608/ejchem.2017.1473.1099.

[16] C. Fernandes, A. Pontes, J. Viana, and A. Gaspar-Cunha, "Modeling and Optimization of the Injection-Molding Process: A Review," Adv. Polym. Technol., Feb. 2016, doi: 10.1002/adv.21683.

[17] ASTM D570-98, "Standard Test Method for Water Absorption of Plastics," ASTM Stand., vol. 98, no. Reapproved 2010, pp. 14, 2010, doi: 10.1520/D0570-98R10E01.2.

[18] M. Kazayawoko, J. J. Balatinecz, and L. M. Matuana, "Surface modification and adhesion mechanisms in woodfiber-

polypropylene composites," J. Mater. Sci., vol. 34, no. 24, pp. 6189-6199, 1999, doi: 10.1023/A:1004790409158. 